\title{
L'EMDR (désensibilisation et retraitement par les mouvements oculaires) comme traitement d'appoint de la dépression unipolaire : une étude contrôlée
}

\author{
Arne Hofmann \\ Institut EMDR allemand, Bergisch Gladbach, Allemagne \\ Angelika Hilgers \\ Pratique libérale, Krefeld, Allemagne \\ Maria Lehnung \\ Pratique libérale, Eckernfoerde, Allemagne \\ Peter Liebermann \\ Pratique libérale, Leverkusen, Allemagne \\ Luca Ostacoli \\ École médicale de l'hôpital Saint Louis Gonzague, Université de Turin, Italie \\ Wolfgang Schneider \\ Académie rhénane de psychothérapie, Allemagne \\ Michael Hase \\ Hôpital Diana, Bad Bevensen, Allemagne
}

\begin{abstract}
La dépression est un trouble mental grave qui constitue un défi pour les systèmes de santé mentale du monde entier. Environ $30 \%$ des patients n'obtiennent pas de rémission complète après le traitement, et plus de $75 \%$ des patients souffrent d'épisodes dépressifs récurrents. Si la psychothérapie et la médication améliorent les taux de rémission, les taux de réussite des traitements actuels sont limités. Dans cette étude exploratoire contrôlée non randomisée, 21 patients souffrant de dépression unipolaire primaire ont été traités avec une moyenne de 44,5 séances de thérapie cognitive comportementale (TCC), avec en moyenne 6,9 séances supplémentaires de désensibilisation et de retraitement par les mouvements oculaires (EMDR). Un groupe contrôle $(n=21)$ a été traité avec une moyenne de 47,1 séances de TCC sans séance EMDR supplémentaire. Le principal moyen de mesure des résultats a été le Questionnaire de Dépression de Beck (BDI-II). Les scores BDI-II des deux groupes étaient identiques avant traitement et les deux traitements ont produit une amélioration significative. Les patients traités avec les séances d'EMDR d'appoint $(p=0,029)$ ont cependant obtenu des améliorations plus importantes. Le nombre de rémissions post-traitement, indiqué par un niveau symptomatique inférieur à 12 sur l'échelle BDI-II, était aussi significativement plus élevé dans le groupe ayant bénéficié de séances d'EMDR d'appoint : ce groupe a présenté davantage de rémissions $(n=18)$ que le groupe de contrôle $(n=8 ; p<0,001)$. Cet effet potentiel de l'EMDR chez les patients souffrant de dépression primaire doit faire l'objet d'études contrôlées randomisées plus larges.
\end{abstract}

Mots-clés : étude contrôlée ; dépression ; désensibilisation et retraitement par les mouvements oculaires (EMDR) ; modèle du traitement adaptatif de l'information (TAI) ; expériences de vie stressantes.

This article originally appeared as Hofmann, A., Hilgers, A., Lehnung, M., Liebermann, P., Ostacoli, L., Schneider, W., \& Hase, M. (2014). Eye Movement Desensitization and Reprocessing as an Adjunctive Treatment of Unipolar Depression: A Controlled Study. Journal of EMDR Practice and Research, 8(3), 103-112. Translated by François Mousnier-Lompré. 
a dépression est un défi sérieux pour les systèmes de santé mentale du monde entier, et c'est un défi qui va en s'aggravant. L'Organisation Mondiale de la Santé a classé la dépression parmi les diagnostics les plus incapacitants. Elle estime qu'elle affecte 340 millions de personnes à travers le monde (Greden, 2001 ; Murray \& Lopez, 1996). Bien qu'un nombre important de patients affectés par la dépression ne connaissent qu'un seul épisode dépressif, une grande partie du poids morbide de la dépression est associée avec la reconnaissance croissante de la nature chronique et récurrente de ce trouble : il a été estimé que $75 \%$ à $90 \%$ des patients ayant traversé un épisode dépressif, selon la longueur de la période d'observation, connaîtront plus d'un épisode dépressif (Angst, 1992 ; Keller, 2002; Kupfer, 1991 ; Maj et coll., 1992). Fait intéressant, un des facteurs de risque majeurs pour la récurrence du trouble réside dans le fait que la rémission du premier épisode soit incomplète (Nierenberg, Petersen \& Alpert, 2003).

\section{Le traitement de la dépression}

Bien que les options de traitement de la dépression se soient élargies au cours des vingt dernières années, l'optimisme accompagnant les nouvelles médications antidépressives telles que les inhibiteurs sélectifs de recapture de la sérotonine (ISRS) est rapidement retombé. En fait, une récente méta-analyse a conclu que les antidépresseurs n'ont qu'un avantage modeste par rapport au placebo, l'ampleur des bénéfices s'accroissant avec la sévérité de la dépression (Fournier et coll., 2010). De plus, l'intervention psychopharmacologique est entravée par les effets secondaires (p.ex. prise de poids) et des problèmes de non-adhésion (Hirschfeld, 2003; Kripalani, Yao \& Haynes, 2007; Reid \& Barbui, 2010).

$\mathrm{Au}$ cours d'un essai randomisé contrôlé (ERC; alternatives de traitement séquencées pour soulager la dépression : STAR ${ }^{\star} D$ [Sequenced Treatment Alternatives to Relieve Depression]), on a utilisé des antidépresseurs (citalopram 20-60 mg) pour traiter 3671 patients atteints de dépression unipolaire. Le taux de rémission initial a été de $37 \%$. En fonction de la réponse des patients au traitement, on a ensuite proposé trois niveaux additionnels de traitement (le niveau 2 étant de la thérapie cognitive comportementale d'appoint [TCC] (Rush et coll., 2006). Le taux de rémission cumulatif après 4 niveaux était de $67 \%$ (la rémission étant définie comme l'absence de symptômes dépressifs mesurée par une échelle d'évaluation standardisée).
Il existe une longue tradition de traitement psychothérapique des dépressions. Une méta-analyse de 28 études a permis de constater qu'une des approches thérapeutiques les plus efficaces dans le domaine du traitement de la dépression, à savoir la thérapie cognitive comportementale, réduisait le taux de rechute de manière significative en comparaison de la pharmacothérapie utilisée seule. Une revue qualitative a conclu que, parmi les patients traités jusqu'à rémission, la thérapie cognitive réduisait la récurrence des rechutes d'environ $50 \%$ (Holon, Steward \& Strunk, 2006). Cependant, les taux de rechute, même chez les patients qui répondent au traitement psychothérapeutique, restaient élevés : en fait, un an après l'interruption d'un traitement psychothérapeutique pour dépression majeure, le taux de rechute était de $29 \%$ et ce dernier augmentait jusqu'à $54 \%$ après deux ans (Vittengl, Clark, Dunn \& Jarrett, 2007).

\section{Stress du quotidien et épisodes dépressifs}

Le stress et ses corrélats neurobiologiques sont des facteurs importants à la fois de cause et de développement des épisodes dépressifs ; les facteurs de stress chroniques et aigus, particulièrement pendant l'enfance, sont bien connus pour contribuer à la maladie et peuvent même déclencher l'apparition de ces troubles (Heim \& Nemeroff, 2001; McFarlane, 2010; Nanni, Uher \& Danese, 2012). Des recherches antérieures ont montré que les premiers épisodes de dépression sont souvent plus étroitement liés à un stress psycho-social particulier que les épisodes suivants ; en fait, les épisodes dépressifs ultérieurs peuvent être déclenchés par des facteurs de stress bien moins importants, voire en l'absence de tout facteur de stress identifiable (Post, 1992).

Une récente méta-analyse d'études génétiques, conduite par Neil Risch et coll. (2009), met en évidence la forte influence des événements stressants de la vie courante tels qu'un danger, une perte ou une humiliation dans le développement de la dépression. Fait intéressant, le seul facteur de risque qui était significativement corrélé avec des épisodes dépressifs était la survenue d'événements stressants. D’après cette méta-analyse, la présence seule d'un polymorphisme du gène transporteur de la sérotonine, même combiné à des événements stressants, n'était pas en corrélation avec l'apparition d'épisodes dépressifs dans la même méta-analyse (Risch et coll., 2009). Ces résultats sont concordants avec les études qui montrent que les événements traumatiques semblent avoir à la fois une réponse dose proche et une relation temporelle avec l'occurrence des épisodes dépressifs 
(Kendler, Hettema, Butera, Gardner \& Prescott, 2003; Teicher, Samson, Polcari \& Andersen, 2009; Wise, Zierler, Krieger $\&$ Harlow, 2001). Au vu des preuves, il semble que les troubles dépressifs soient davantage en rapport avec les troubles liés au stress et au traumatisme que ne le reflètent les approches actuelles de la dépression (Horwitz \& Wakefield, 2007 ; Maj, 2012).

Le trouble typique des troubles liés aux traumas (ou, si l'on utilise la version beta de la $11^{\mathrm{e}}$ révision de la Classification Internationale des Maladies [CIM-11], des troubles spécifiquement liés au stress) est l'état de stress post-traumatique (ESPT). L'ESPT est un trouble bien étudié dont le traitement s'est amélioré de façon significative au cours des 20 dernières années. Des études portant sur les approches du traitement de l'ESPT ont montré que les traitements s'adressant spécifiquement au trauma améliorent significativement plus les symptômes de l'ESPT qu'une approche thérapeutique non spécifique (Bisson et coll., 2007; Bisson, Roberts, Andrew, Cooper \& Lewis, 2013).

Fait intéressant, approximativement $80 \%$ des patients atteints d'ESPT souffrent également d'une comorbidité importante, en particulier la dépression. Cette dépression comorbide tend à s'améliorer nettement si l'ESPT seul est traité en premier, sans aucun traitement spécifique de la dépression (Ho \& Lee, 2012 ; Van Etten \& Taylor, 1998). Néanmoins, les méthodes de traitement spécifique du traumatisme qui sont capables de traiter les souvenirs stressants à la source de l'ESPT sont aujourd'hui rarement étudiées pour le traitement des troubles dépressifs primaires (Grey, 2011).

\section{L'EMDR}

La désensibilisation et le retraitement par les mouvements oculaires (EMDR) est une approche psychothérapeutique en huit phases qui a été développée par Francine Shapiro (2001). Un des composants-clés de l'EMDR est la stimulation bilatérale (avec, par exemple, les mouvements oculaires), effectuée pendant que les patients se concentrent sur le souvenir qui cause leurs symptômes actuels. L'EMDR est l'une des méthodes psychothérapeutiques les plus efficaces pour le traitement de l'ESPT (Bisson et coll., 2013). Certaines études ont suggéré que l'EMDR peut être plus rapide que d'autres traitements efficaces (cf. Van Etten \& Taylor, 1998). Les effets du traitement EMDR semblent stables dans la durée, selon une étude de suivi contrôlée après 35 mois (Hoegberg et coll., 2007).

La thérapie EMDR s'appuie sur un modèle du traitement de l'information connu sous le nom de modèle de traitement adaptatif de l'information (TAI) (Shapiro, 2001). Une des hypothèses essentielles du modèle TAI est que les souvenirs (perturbants) stockés de façon dysfonctionnelle sont la cause de plusieurs pathologies mentales, comprenant l'ESPT, d'autres troubles d'origine traumatique, de même que certains troubles dépressifs et anxieux. L'EMDR est actuellement utilisée pour traiter toute la gamme des plaintes qui font suite à des expériences de vie perturbatrices (Shapiro \& Maxfield, 2002).

\section{L'EMDR dans le traitement des troubles dépressifs}

Développée à l'origine pour soulager la détresse causée par les souvenirs traumatiques, en particulier ceux associés à un ESPT, la thérapie EMDR a rapidement été proposée pour le traitement d'autres pathologies, qui ne sont pas nécessairement reliées à des événements traumatiques remplissant le critère A de l'ESPT. En fait, l'EMDR était déjà utilisée par les cliniciens au début des années 1990 pour le traitement de patients souffrant de dépression (Marquis, 1991). Des études systématiques ont démontré les effets de l'EMDR sur la dépression liée à l'ESPT. Dans un essai clinique randomisé, Van der Kolk et coll. (2007) ont comparé l'efficacité du traitement à la fluoxétine par rapport à l'EMDR et à un placebo sur une population souffrant d'ESPT. Après l'intervention, le groupe traité avec l'EMDR montrait un score BDI-II significativement plus bas que le groupe traité par la fluoxétine. Une récente méta-analyse sur le traitement de l'ESPT et de la dépression comorbide fait écho à ces résultats : Ho et Lee (2012) ont montré que l'EMDR semblait avoir un effet significativement plus important sur la dépression comorbide que la TCC, bien que l'effet sur l'ESPT fût similaire.

Cette baisse des symptômes dépressifs, à la suite du traitement EMDR de souvenirs que les patients ressentent comme traumatiques, semble ne pas être limitée aux seuls patients atteints d'ESPT. Dans une étude contrôlée, Wilson, Becker \& Tinker (1995) ont traité un groupe souffrant de souvenirs stressants. Si seulement $54 \%$ de ces patients remplissaient les critères pour un ESPT ( $\mathrm{y}$ compris le critère $\mathrm{A}$ définissant l'événement comme traumatique), tous tirèrent bénéfice du traitement EMDR, ainsi que le prouvèrent les améliorations significatives de leur ESPT et de leurs symptômes dépressifs. Un suivi à 15 mois montrait que les deux effets bénéfiques se maintenaient (Wilson et coll., 1995, 1997).

La première série de cas portant sur deux adolescents souffrant de dépression majeure et traités avec 
l'EMDR fut publiée en 2008. La réussite de leur traitement a requis, respectivement, 3 et 7 séances, et un suivi à 3 mois montrait que les résultats positifs obtenus se maintenaient. Dans les deux cas, l'EMDR a été utilisé avec succès dans le traitement d'événements liés à une perte ou à une modification de relations, mais ne remplissant pas le critère de catégorie A de l'ESPT. Ils pouvaient plutôt être considérés comme des événements de vie stressants ou des « traumas d'attachement ». Le traitement améliora la dépression de manière significative dans les deux cas et eut un effet positif à la fois sur le traitement émotionnel-cognitif et sur l'organisation conceptuelle de la mémoire à long terme (Uribe, Ramirez \& Mena, 2010).

Des événements tels que ceux que l'on vient de citer semblent être des facteurs de risque spécifiques favorisant l'émergence de troubles dépressifs. Dans une large étude rétrospective, des événements liés à des pertes, à des séparations ou à des humiliations étaient liés de manière significative avec des épisodes dépressifs un mois plus tard (Kendler et coll., 2003).

L'observation que les symptômes dépressifs sont plutôt liés à des événements ne remplissant pas le critère A est également évidente dans plusieurs rapports de cas, où des patients étaient traités avec succès par l'EMDR et où l'EMDR était utilisée, soit comme modalité thérapeutique unique, soit administrée en association avec d'autres approches thérapeutiques (Broad \& Wheeler, 2006 ; Grey, 2011 ; Manfield, 1998 ; Shapiro, 2009 ; Shapiro \& Silk Forrest, 1997 ; Sun, Wu \& Chiu, 2004 ; Tinker \& Wilson, 1999).

Malheureusement, aucune étude contrôlée n'a été publiée jusqu'ici concernant l'EMDR comme intervention pour des patients ayant pour seul diagnostic la dépression. L'objectif de notre étude contrôlée était donc de commencer à combler ce manque et d'explorer le potentiel d'un traitement EMDR d'appoint chez des patients souffrant de dépression primaire.

\section{Méthode}

En Allemagne, en raison du système d'assurance, les traitements psychothérapeutiques sont limités à l'application de trois orientations psychothérapeutiques générales : la psychothérapie psychodynamique, la psychothérapie psychanalytique et la TCC. Au sein de ces orientations de base, certaines méthodes psychothérapeutiques additionnelles sont permises. En 2006, le conseil scientifique allemand pour la psychothérapie a reconnu l'EMDR comme une méthode psychothérapeutique scientifiquement prouvée pour le traitement de l'ESPT (Conseil Scientifique [wissenschaftlicher Beirat] de la Psychothérapie,
2006). Ainsi, la plupart des psychothérapeutes allemands qui sont formés à l'EMDR l'intègrent à leur approche de traitement psychothérapeutique habituelle.

\section{Procédure}

Tous les patients de cette étude souffraient d'une crise dépressive unipolaire et ont été traités à la clinique ambulatoire de l'Académie rhénane de psychothérapie (désignée ici comme RHAP : Rhineland Academy for Psychotherapy), un centre de formation en TCC sis à Krefeld (Allemagne). Le traitement standard pour la dépression dans cette clinique est la TCC. Si nécessaire, un traitement médicamenteux est prescrit, lors de séances séparées, par un psychiatre indépendant. Entre 2008 et 2012, certains thérapeutes en dernière année de formation en TCC à la RHAP ont également reçu une formation de base en l'EMDR, suivie d'une supervision de longue durée.

Les patients qui souffraient de dépression unipolaire et qui avaient été assignés par hasard à ces thérapeutes formés à l'EMDR se sont vu offrir l'opportunité de participer à la présente étude. Nous avons recruté un groupe de 30 patients qui ont accepté de donner leur consentement éclairé pour être traités avec des séances EMDR d'appoint, incluses dans leur traitement TCC (traitement habituel [TH] + EMDR).

Dès le début de l'étude, pour chaque patient qui commençait le traitement EMDR, un patient TH était choisi de façon aléatoire parmi les patients de la même clinique qui remplissaient les critères inclusion/exclusion et qui avaient reçu le traitement TCC. Les thérapeutes TH achevaient à l'institut leur formation TCC au cours de la même période que les thérapeutes EMDR, mais ils ne recevaient pas de formation EMDR.

\section{Les participants}

Les participants $T H+E M D R$. Les critères d'inclusion pour les participants TH + EMDR étaient la capacité de suivre une psychothérapie et la volonté de participer à des séances EMDR qui allaient travailler sur les souvenirs stressants qu'on pensait en rapport avec le(s) épisode(s) dépressif(s). Les critères d'exclusion étaient une comorbidité accompagnée d'autres troubles psychologiques sévères, des troubles psychotiques ou un ESPT. On comptait aussi parmi les critères d'exclusion les difficultés cognitives importantes, les maladies physiques graves nécessitant une intervention chirurgicale et les procédures judiciaires en cours. À cause du caractère exploratoire de l'étude, six patients ont été acceptés dans le groupe d'étude 
malgré le fait qu'ils répondaient au critère de trouble additionnel. Dans le groupe de contrôle, deux patients présentant un diagnostic comorbide ont été acceptés. Les comorbidités du groupe TH + EMDR comprenaient un trouble panique (deux), une phobie sociale, un trouble borderline de la personnalité (deux) et un trouble alimentaire non spécifié. Les comorbidités du groupe TH étaient la consommation de cannabis et l'abus d'alcool, chacun dans un cas.

L'échantillon initial TH + EMDR était composé de 45 patients externes de la clinique psychothérapique de la RHAP qui avaient un diagnostic de dépression unipolaire et avaient été assignés à un des thérapeutes du groupe formé à l'EMDR. Parmi ces patients, quinze ont été exclus de l'étude : dix d'entre eux ne répondaient pas aux critères d'inclusion et cinq autres ont refusé de participer. Le groupe de 30 patients restant a ensuite été suivi tout au long du traitement ; 21 d'entre eux ont reçu le traitement d'appoint complet et leurs scores ont été analysés. Parmi les 9 patients qui n’ont pas reçu le traitement d'appoint complet, l'un d'eux refusa d'avoir d'autres séances d'EMDR, et les 8 autres n'ont pas reçu le traitement complet pour d'autres raisons. Trois d'entre eux souffraient d'un trouble comorbide : trouble alimentaire non spécifié, phobie sociale et un trouble borderline. Parmi les 9 patients qui ont abandonné le traitement EMDR, 8 ont mené à terme leur traitement TH, un seul n'est pas allé jusqu'au bout.

Les participants $T H$. Les critères d'inclusion pour les participants TH étaient les mêmes que pour le participants TH + EMDR (comorbidité associée à d'autres troubles psychologiques sévères, troubles psychotiques, ESPT, déficiences cognitives profondes, maladie physique grave nécessitant une opération ou procédure judiciaire en cours). Les critères d'inclusion comprenaient le fait d'avoir mené à terme le programme $\mathrm{TH}$ et de remplir les critères d'inclusion de l'étude (un diagnostic de dépression unipolaire et l'aptitude à suivre une psychothérapie).

Les patients TH avaient été traités par d'autres thérapeutes de la clinique non formés à l'EMDR. Afin de les faire correspondre au 21 patients ayant complété le traitement TH + EMDR, les informations concernant 21 patients qui avaient terminé leur traitement TH ont été sélectionnées de façon aléatoire pour l'analyse finale de ce groupe contrôle TH.

\section{Le traitement}

Le point final du traitement TH + EMDR comme du traitement TH était déterminé par le thérapeute (ainsi que par les superviseurs) et le patient lui-même sur des critères cliniques uniquement. On a considéré que le nombre minimum de séances pour une " thérapie EMDR d'appoint » était de 3 séances.

Les thérapeutes. Tous les thérapeutes participant à l'étude étaient des étudiants en psychothérapie en formation TCC avancée. Les 14 thérapeutes EMDR ont terminé avant l'étude une formation EMDR approuvée par l'association internationale d'EMDR (EMDRIA). Dans la mesure où un candidat ne peut avoir qu'un nombre limité de patients pendant sa formation, chacun d'entre eux se voyait adresser au plus un à quatre patients qu'il pouvait traiter pendant l'étude.

La fidélité $d u$ traitement. Tous les thérapeutes de l'étude (groupe TH + EMDR et groupe TH) étaient régulièrement suivis en supervision TCC afin de s'assurer de la conformité de leurs traitements TCC. Cette supervision avait systématiquement lieu toutes les quatre séances de thérapie. Les thérapeutes du groupe TH + EMDR étaient régulièrement soumis à une supervision additionnelle conduite par un formateur EMDR expérimenté afin de s'assurer la fidélité au traitement EMDR et sa concordance avec les standards d'EMDR Europe.

Le traitement TCC. Le traitement TCC s'est conformé aux manuels de thérapie cognitive pour le traitement de la dépression (Beck, Rush, Shaw \& Emery, 1979 ; Hautzinger, 2003). La thérapie travaille systématiquement sur les croyances dysfonctionnelles et enseigne des techniques de contrôle des affects négatifs et de leurs influences sur les sentiments et le comportement. En outre, on enseigne aux patients à prendre des décisions et à augmenter la fréquence et la qualité des expériences agréables. L'intégralité du traitement TCC de notre étude était conduite en séances individuelles, en face-à-face. Du travail personnel à faire hors séance aide les patients à améliorer leurs capacités, par exemple leurs compétences sociales dans la vie quotidienne.

Le traitement EMDR. Le traitement EMDR a suivi le plan en huit phases de l'EMDR décrit par Shapiro (2001). Les cibles EMDR étaient sélectionnées sur le modèle TAI, qui recherche les événements stressants liés à la dépression. Selon les besoins spécifiques des patients, on a employé l'EMDR afin de traiter les événements traumatiques (critère $A$ ) ou non traumatiques (ne répondant pas au critère $A$ ) qui coïncidaient avec l'épisode dépressif actuel ou avaient un possible lien avec celui-ci (" événements déclencheurs de l'épisode »). Au cours d'études antérieures, une approche systématique a été développée ; elle a récemment fait 
l'objet d'une publication et fait partie d'une étude ERC en cours (Hofmann et coll., 2014).

\section{Mesures}

Le diagnostic de dépression était établi lors d'un entretien initial à l'aide du Structured Clinical Interview for DSM-IV Axis-I Disorders (Wittchen, Zaudig \& Wunderlich, 1997). L’entretien n'était pas mené en aveugle, mais conduit par le thérapeute qui suivrait plus tard le patient. Le résultat principal de l'étude était le niveau des symptômes dépressifs tels que mesurés par le Questionnaire de Dépression de Beck II (BDI-II ; Beck, Steer \& Brown, 1996 ; Hautzinger, Keller \& Kühner, 2006). Le BDI-II est un instrument d'évaluation autoadministré en 21 points avec de bonnes propriétés psychométriques. Il possède une grande sensibilité aux changements pendant la thérapie, ce qui en faisait un outil utile pour notre étude. Les scores vont de 0 à 63 , avec les variations suivantes : 0-13 : écart minimal, 14-19 : dépression légère, 20-28: dépression modérée et 29-63: dépression grave. On procéda au test en pré- et post-traitement.

\section{Analyse des données}

Nous avons enregistré les scores BDI-II avant (BDI-II pré) et après (BDI-II post) traitement. L'analyse des données a été faite par une analyse de variance par mesures répétées (ANOVA), avec le traitement (TCC ou TCC + EMDR) comme facteur entre groupes et le moment de la mesure (BDI-pré ou BDI-post) comme facteur entre sujets. Nous avons utilisé le niveau alpha conventionnel de $5 \%$ (bilatéral). Les tests $t$ post hoc prévus ont été menés pour approfondir l'examen des différences entre les notes au prétraitement et au posttraitement à l'intérieur des groupes et entre eux.
Nous avons également enregistré la rémission de la dépression (rémission de l'épisode/pas de rémission) en prenant comme seuil un score BDI post de 12 . Ces données ne répondant pas aux critères d'une analyse paramétrique, nous avons utilisé le test $U$ non paramétrique de Mann-Whitney pour les échantillons indépendants, $\alpha=0,05$. Les données ont été analysées avec un IBM SPSS Statistics 22.

\section{Résultats}

L'âge moyen des 42 patients $(21+21)$ était de 40,38 ans $(\sigma=10.38)$. L'âge moyen du groupe $\mathrm{TH}$ était de 40,67 ans $(\sigma=12,145)$; l'âge moyen du groupe TH + EMDR était de 40,1 ans $(\sigma=9,859)$. Les différences d'âge entre les deux conditions de traitement n'étaient pas statistiquement significatives à $\alpha=0,05$. De même, la différence de sexe entre les deux groupes n'était pas significative. 11 des 21 patients du groupe TH + EMDR et 15 des 21 patients du groupe TH souffraient d'épisodes dépressifs récurrents.

Les deux groupes ne différaient pas par rapport à la gravité de la dépression (F3x.1/F3x.2) ni par rapport à la chronicité (F32.x/F33.x). Les résultats de l'analyse statistique des paramètres des participants sont montrés dans le Tableau 1.

Les patients $\mathrm{TH}+$ EMDR ont eu en moyenne 6,9 séances d'EMDR (écart $=3-16$ séances) et suivi une moyenne de 37,58 séances de TCC, pour une moyenne totale de 44,48 séances de thérapie $(\sigma=11,48)$. Le groupe $\mathrm{TH}$ a eu une moyenne de 47,11 séances de TCC $(\sigma=7,41)$. Un test $t$ d'indépendance des échantillons n'a pas révélé de différence statistique significative entre les nombres moyens de séances $(t[28]=-0,631, p=0,533)$.

TABLEAU 1. Caractéristiques de l'échantillon

\begin{tabular}{|c|c|c|c|}
\hline & Groupe TH $+\operatorname{EMDR}(n=21)$ & Groupe TH $(n=21)$ & Statistiques \\
\hline \multirow[t]{2}{*}{ Âge (années) } & $40,1(\sigma 9,67)$ & $40,67(\sigma 12,145)$ & $t=-0,125(\mathrm{df} 28)$ \\
\hline & & & $p=0,903(\mathrm{~ns})$ \\
\hline \multirow[t]{2}{*}{ Homme/femme } & $4 / 17$ & $5 / 16$ & $U=-0,372$ \\
\hline & & & $p=0,710(\mathrm{~ns})$ \\
\hline Gravité de l'épisode & $1 / 18 / 2$ & $0 / 21 / 0$ & $\chi^{2}=0,350$ \\
\hline F3X.0/3X.1/3X.2 & & & $p=0,573(\mathrm{~ns})$ \\
\hline \multirow[t]{2}{*}{ Dépression récurrente (F33.x) } & 11 & 15 & $\chi^{2}=0,000$ \\
\hline & & & $p=1,000(\mathrm{~ns})$ \\
\hline
\end{tabular}

Note. $\mathrm{TH}=$ traitement habituel $; \mathrm{EMDR}=$ désensibilisation et retraitement par les mouvements oculaires 


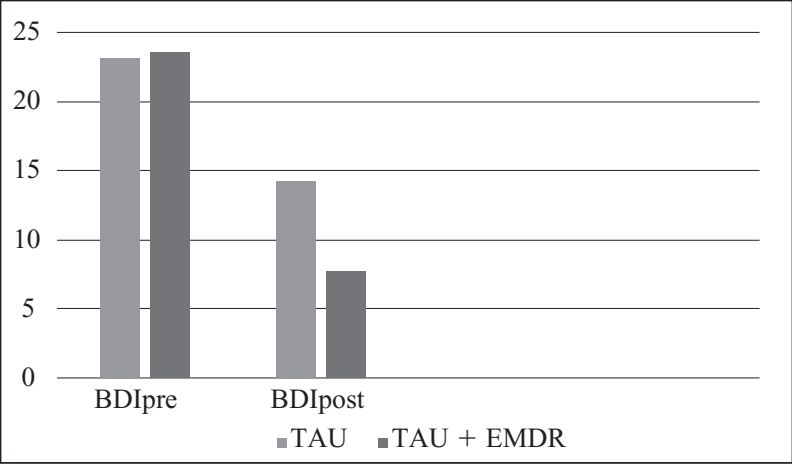

FIGURE 1. Notes au BDI-II selon les deux conditions de traitement.

Note. BDIpre $=$ note au Questionnaire de Dépression de Beck au prétraitement; BDIpost = note au Questionnaire de Dépression de Beck au post-traitement ; TAU = traitement habituel ; EMDR = désensibilisation et retraitement par les mouvements oculaires

\section{Analyse des effets du traitement}

L'analyse de données par mesures répétées ANOVA a révélé un effet d'interaction significatif. Une comparaison entre les résultats des deux groupes de traitement a montré une interaction significative du traitement et de la durée, démontrant une différence significative entre les deux traitements $(F[1,40]=$ $5,108, p=0,029)$, indiquant que les patients du groupe $\mathrm{TH}+$ EMDR avaient un pattern de changement différent du groupe TH. Des tests post hoc ont été effectués pour mieux évaluer ces différences (Figure 1).

Un test $t$ post hoc a été mené afin de comparer les résultats BDI-II en prétraitement. Aucune différence significative n'a été relevée $(t[40]=0,149$, $p=0,882)$, indiquant que les participants des groupes $\mathrm{TH}+$ EMDR et TH ne présentaient pas de différence sur le plan de la gravité de leurs scores BDI-II en prétraitement (Tableau 2 et Figure 1). Des tests t post hoc ont été effectués pour déterminer si les deux traitements avaient produit une diminution significative des scores BDI-II. Les résultats montrèrent des différences importantes pour le groupe TH + EMDR $(t[20]=6,604$, $p=0,000)$ ainsi que pour le groupe $\mathrm{TH}(t[20]=6,886$, $p=0,000):$ les deux traitements réduisaient donc efficacement les symptômes de la dépression.

Un test $\mathrm{t}$ a été conduit afin de comparer les scores BDI-II en post-traitement. Les résultats ont montré que les deux traitements différaient significativement l'un de l'autre : $(t[40]=-2,675, p=0,011)$, indiquant que les symptômes des participants du groupe $\mathrm{TH}+$ EMDR montraient une amélioration plus importante de leur dépression que les patients TH traités par TCC uniquement.
TABLEAU 2. Résultats

\begin{tabular}{lcc}
\hline & $\begin{array}{c}\text { Groupe TH + EMDR } \\
(n=21)\end{array}$ & $\begin{array}{c}\text { Groupe TH } \\
(n=21)\end{array}$ \\
\hline $\begin{array}{c}\text { Résultat BDI-II en } \\
\text { prétraitement }\end{array}$ & $23,57(\sigma 7,639)$ & $23,19(\sigma 8,892)$ \\
$\begin{array}{c}\text { Résultat BDI-II en } \\
\text { post-traitement }\end{array}$ & $7,86(\sigma 5,452)$ & $14,24(\sigma 9,476)$ \\
$\begin{array}{c}\text { Nombre de } \\
\text { rémissions }\end{array}$ & 18 & 8 \\
\hline
\end{tabular}

Note. Une rémission est définie par un score BDI-II (Questionnaire de Dépression de Beck) de 12 ou moins en post-traitement ; $\mathrm{TH}=$ Traitement habituel $; \mathrm{EMDR}=$ Désensibilisation et retraitement par les mouvements oculaires.

Les résultats de la comparaison des rémissions, définies par un résultat BDI-II égal ou inférieur à 12, différaient selon les catégories de traitement, comme l'a montré le test $U$ de Mann-Whitney $(U=105,000$; $p<0,001)$, le groupe TH + EMDR montrant significativement plus de rémissions $(n=18)$ que le groupe TH $(n=8)$ (Tableau 2).

Médication. Six patients du groupe TH ont reçu des antidépresseurs au début de la psychothérapie contre neuf patients dans le groupe TH + EMDR. Des tests de $\chi^{2}$ n'ont révélé aucune différence significative. Au cours de l'étude, le psychiatre a recommandé quatre changements de médication dans le groupe $\mathrm{TH}+$ EMDR et six dans le groupe $\mathrm{TH}$.

Tous les changements de traitement médicamenteux dans le groupe TH étaient de premières prescriptions d'antidépresseurs ; on a observé une première prescription d'antidépresseurs dans le groupe $\mathrm{TH}+$ EMDR. Les types d'antidépresseurs et leur distribution en début de psychothérapie sont donnés dans le Tableau 3.

\section{Discussion}

Cette étude exploratoire visait à déterminer l'efficacité clinique de séances EMDR d'appoint chez des patients atteints de dépression unipolaire sans ESPT.

TABLEAU 3. Types d'antidépresseurs

\begin{tabular}{lcccc}
\hline & ISRS & ANSS & Autre & Aucun \\
\hline TH + EMDR & 5 & 0 & 4 & 12 \\
TH & 1 & 1 & 4 & 15 \\
\hline
\end{tabular}

Note. ISRS = inhibiteurs sélectifs de recapture de la sérotonine ; ANSS = antidépresseurs noradrénergiques et sérotoninergiques spécifiques ; $\mathrm{TH}=$ traitement habituel $; \mathrm{EMDR}=$ désensibilisation et retraitement par les mouvements oculaires 
Elle comprenait deux groupes de 21 patients $(n=42)$ dont l'âge, le sexe et la chronicité de leur dépression étaient en correspondance. Les deux groupes ont été traités avec, en moyenne, 45,7 séances de TCC. Un groupe a été traité avec 6,9 séances d'EMDR (nombre de séances compris entre 3 et 16) comprises dans les 45,7 séances. L'autre groupe fut traité avec un nombre similaire de séances de TCC uniquement.

L'étude a révélé principalement une différence significative dans la diminution des scores BDI-II après traitement, démontrant que les patients avaient tiré profit de la TCC et de la TCC avec le traitement EMDR d'appoint. Les résultats montraient aussi une diminution plus importante des scores BDI-II pour le groupe $\mathrm{TH}+$ EMDR que pour le traitement TCC seul $(p=0,011)$. Bien que la TCC soit considérée depuis de nombreuses années comme un traitement très efficace et bien établi de la dépression, les séances EMDR supplémentaires peuvent augmenter l'effet bénéfique du traitement de la dépression.

Une analyse plus approfondie de nos groupes a montré que le nombre de rémissions de la dépression (correspondant à un niveau de symptôme de 12 ou moins au BDI-II) indiquait une différence très significative, prouvant les bénéfices supplémentaires tirés par le groupe qui avait reçu des séances d'EMDR $(p<0,001)$. Considérant qu'aujourd'hui, de nombreux patients répondent mal à un traitement adapté par antidépresseurs et/ou à la psychothérapie, et que plus de $30 \%$ ne parviennent pas à une rémission complète quel que soit le type de traitement, les résultats de l'ajout d'une thérapie EMDR que l'on observe dans cette étude méritent d'être rapportés et devraient faire l'objet d'études contrôlées plus larges. Dans la mesure où les patients qui n'obtiennent pas de rémission complète après traitement ont un risque plus élevé de rechute, le traitement EMDR d'appoint pourrait également évoluer et devenir un outil supplémentaire de prévention des rechutes de dépression (Nierenberg et coll., 2003).

Cette étude étant la première à utiliser l'EMDR dans le traitement de la dépression primaire, il est intéressant de noter qu'un nombre limité de séances EMDR, en moyenne 6 ou 7, intégrées aux 45,7 séances de psychothérapie, semble avoir fait une différence significative sur le plan de la symptomatologie des patients. Une des explications de ce résultat pourrait être non seulement que des événements stressants significatifs peuvent contribuer au déclenchement d'un épisode dépressif, mais que les souvenirs de ces événements peuvent également contribuer à maintenir ensuite la dépression. Ainsi, le traitement par l'EMDR des souvenirs de ces événements stressants, stockés de façon dysfonctionnelle, chez ces patients souffrant de dépression primaire, peut avoir joué un rôle dans l'amélioration significative des symptômes dans le groupe EMDR. Cela peut également avoir contribué au nombre significativement plus élevé des rémissions chez les patients de ce groupe. Cette étude pourrait encore être considérée comme un encouragement à faire bénéficier les patients dépressifs, dont le traitement nécessite souvent plusieurs interventions, de l'inclusion de l'EMDR en tant que thérapie d'appoint en plus des autres approches thérapeutiques employées dans leur traitement.

Notre étude pourrait également être considérée comme une validation de la sélection par Bae et ses collaborateurs (2008) des souvenirs qu'ils ont ciblés en EMDR dans leurs deux cas de dépression chez l'adolescent. Dans chacun de ces deux cas, ils n'avaient en effet pas pris pour cible des événements remplissant le critère $\mathrm{A}$, mais d'importants événements traumatiques de l'ordre de l'attachement. Dans notre étude, ces " événements d'attachement " étaient également ceux sur lesquels se sont concentrés les thérapeutes dans la plupart des séances d'EMDR. Parmi les 21 patients qui ont reçu de l'EMDR dans notre groupe d'étude, seulement 5 avaient rapporté des événements répondant au critère $A$, qui furent alors traités avec l'EMDR (trois accidents de la route, un viol et un cas de violence domestique). Les autres patients avaient rapporté des souvenirs stressants qui ne remplissaient pas le critère A d'ESPT, mais qui furent également traités avec l'EMDR ; la plupart de ces souvenirs décrivaient des événements de type relationnel qui étaient toujours perturbants pour eux. L'un d'entre eux concernait le diagnostic d'une récidive de cancer. Beaucoup d'autres concernaient des pertes, des séparations, ou encore des humiliations, c'est-à-dire tout à fait le type de souvenirs qui, selon l'étude de Kendler et ses collaborateurs (2003), sont en rapport avec l'apparition d'épisodes dépressifs.

Le modèle EMDR du TAI postule que les événements stressants du quotidien, pour se retrouver "stockés de façon dysfonctionnelle au sein des réseaux mnésiques » et générer la pathologie présente, n'ont pas besoin d'être de l'ordre d'un danger vital (critère A). Généralement, pour ce type de souvenirs dysfonctionnels, l'événement passé est toujours vécu comme perturbant par le patient dans le présent. Cela s'accorde bien avec les résultats d'une étude qui montrent que les victimes d'événements stressants de la vie courante ne décrivent pas les événements répondant au critère A comme étant " plus traumatisants " que d'autres événements stressants (Gold, Marx, Soler-Baillo \& Sloan, 2005). 
L'EMDR est souvent considérée comme une méthode permettant de traiter seulement l'ESPT ; on comprend alors ce point comme une limitation : les événements qu'on peut cibler en EMDR doivent forcément être des événements répondant au critère A. Au moins dans le cas de la dépression, il ne semble pas en être ainsi : les traumas d'attachement, comme la perte, les séparations ou les humiliations semblent davantage en rapport avec le développement d'un épisode dépressif que les événements du critère A (Kendler \& coll., 2003), et le traitement en EMDR de ce type de souvenirs semble améliorer les symptômes dépressifs.

D’après ces études et les résultats de notre étude sur des patients dépressifs, il se pourrait bien que dans le cas de la dépression, il ne soit pas si important qu'un souvenir à traiter soit " traumatisant » (au sens du critère A de l'ESPT), mais plutôt qu'il soit encore stocké de façon dysfonctionnelle, au sens où l'entend le modèle TAI, et qu'il continue de produire une certaine pathologie psychobiologique (production d'intrusions ou d'un sentiment subjectif de détresse lorsque le patient se rappelle le souvenir). Toutefois, cela ne pourra être testé que dans d'autres études systématiques.

Les limitations de cette étude empêchant la généralisation des résultats sont de type méthodologique, telles que le manque de randomisation, le faible nombre de patients, le manque d'évaluations indépendantes et l'utilisation d'un instrument d'évaluation auto-administré en tant que résultat. Une autre limitation tient à ce que, dans notre étude, nous avons sélectionné un groupe de contrôle de patients ayant mené leur thérapie TCC à son terme dans la même clinique et en même temps, et non un groupe qu'on aurait randomisé et suivi tout au long du traitement. Le fait que cette étude concerne des patients ayant mené à terme leur thérapie, et qu'elle a pu laisser de côté des cas de dépression plus complexes avant l'analyse, peut provoquer une surestimation des effets de l'EMDR. Parmi les 30 patients TH + EMDR, 9 n'ont pas terminé le traitement EMDR et ont été perdus pour l'analyse. Trois de ces patients étaient parmi les six présentant des aspects comorbides graves (un patient avec une phobie sociale, un avec des troubles alimentaires non spécifiés et un des deux patients de l'étude qui souffraient d'un trouble borderline). Le patient qui avait refusé d'autres séances d'EMDR décrivait une augmentation du stress lors des séances d'EMDR, ce qui l'avait découragé de poursuivre (il ne faisait pas partie des cas de comorbidité). Dans les deux groupes de notre étude, la courte expérience clinique de nos thérapeutes a pu également jouer un rôle et restreindre la possibilité de généraliser les conclusions de l'étude. En revanche, le potentiel de l'EMDR d'appoint se voit dans le cas des deux patients borderline de notre étude. Ils ont reçu tous deux 45 séances de psychothérapie. Bien que l'un d'entre eux n'ait eu que deux séances d'EMDR et n'ait pas montré d'amélioration à la fin de l'étude, l'autre patient a eu 13 séances d'EMDR et a terminé sa thérapie avec une amélioration significative et un score BDI-II de 10.

La dernière limitation réside dans l'absence actuelle d'information sur le suivi des patients. C'est là l'une des limites organisationnelles de notre étude. Nous espérons pouvoir y remédier par un essai multicentrique contrôlé sur l'EMDR chez des patients atteints de dépression, essai qui a déjà commencé et qui fera l'objet d'un suivi.

En dépit des limitations de cette étude, nous estimons que cette première étude contrôlée sur l'emploi de l'EMDR chez des patients dépressifs peut encourager le lancement d'autres études dans ce domaine. Il peut être intéressant qu'une technique telle que l'EMDR, qui traite les souvenirs stressants, puisse s'ajouter aux options thérapeutiques proposées et aider d’autres patients dépressifs à obtenir une rémission complète de leur épisode dépressif.

\section{Implications pour de futures recherches}

Les recherches à venir sur l'emploi de l'EMDR dans le traitement de patients dépressifs devraient se concentrer sur des études contrôlées randomisées. Elles pourraient étudier l'intégration de l'EMDR à d'autres traitements, et la comparer à son utilisation seule pour différents sous-groupes de troubles dépressifs.

Les recherches pourraient évaluer quels types de patients présentant des troubles dépressifs bénéficieraient le plus de la thérapie EMDR. Étant donnée le rapport entre rémissions partielles et épisodes récurrents, il est possible que les patients dépressifs susceptibles de bénéficier le plus d'études futures sur l'EMDR soient ceux qui souffrent de dépressions récurrentes. Compte tenu de la connexion possible entre la persistance des symptômes dépressifs et des souvenirs stockés de façon dysfonctionnelle et la capacité de l'EMDR à traiter ceux-ci, certains groupes de patients dépressifs chroniques pourraient également tirer avantage de futures études sur l'EMDR. De même, des recherches concernant la valeur de l'EMDR d'appoint pour les enfants et les adolescents au début de leur "parcours dépressif " pourraient être utiles. Elles permettraient également d'évaluer si l'EMDR peut apporter un plus grand bénéfice à ces enfants et ces adolescents souffrant de dépression 
et ayant vécu des événements traumatiques ou des pertes. Une étude comparative du rapport coût-efficacité pourrait également évaluer les bénéfices potentiels de ces interventions par rapport à des ressources médicales limitées.

\section{Bibliographie}

Angst, J. (1992). How recurrent and predictable is depressive illness. In S. Montgommery \& F. Roullon (Eds.), Long-term treatment of depression: Perspectives in psychiatry (Vol. 3, pp. 1-13). Chichester, United Kingdom: Wiley.

Bae, H., Kim, D., \& Park, J. C. (2008). Eye movement desensitization and reprocessing for adolescent depression. Psychiatry Investigation, 5, 60-65.

Beck, A. T., Rush, A. J., Shaw, B. F., \& Emery, G. (1979). Cognitive therapy of depression. New York, NY: Guilford.

Beck, A. T., Steer, R. A., \& Brown, G. K. (1996). Beck Depression Inventory-II (BDI-II). San Antonio, TX: Harcourt Assessment.

Bisson, J. I., Ehlers, A., Matthews, R., Pilling, S., Richards, D., \& Turner, S. (2007). Psychological treatments for chronic post-traumatic stress disorder. Systematic review and meta-analysis. British Journal of Psychiatry, 190, 97-104.

Bisson, J. I., Roberts, N. P., Andrew, M., Cooper, R., \& Lewis, C. (2013). Psychological therapies for chronic post-traumatic stress disorder (PTSD) in adults. Cochrane Database of Systematic Reviews, (12), CD003388.

Broad, R. D., \& Wheeler, K. (2006). An adult with childhood medical trauma treated with psychoanalytic psychotherapy and EMDR: A case study. Perspectives in Psychiatric Care, 42, 95-105.

Fournier, J. C., DeRubeis, R. J., Hollon, S. D., Dimidjian, S., Amsterdam, J. D., Shelton, R. C., \& Fawcett, J. (2010). Antidepressant drug effects and depression severity: A patient-level meta-analysis. Journal of the American Medical Association, 303 (1), 47-53.

Gold, S. D., Marx, B. P., Soler-Baillo, J. M., \& Sloan, D. M. (2005). Is life stress more traumatic than traumatic stress? Journal of Anxiety Disorders, 19, 687-698.

Greden, J. F. (2001). The burden of recurrent depression: Causes, consequences, and future prospects. Journal of Clinical Psychiatry, 62 (Suppl. 22), 5-9.

Grey, E. (2011). A pilot study of concentrated EMDR: A brief report. Journal of EMDR Practice and Research, 5, 14-24.

Hautzinger, M. (2003). Kognitive Verhaltenstherapie bei Depressionen. Weinheim, Allemagne: Beltz.

Hautzinger, M., Keller, F., \& Kühner, C. (2006). Beck Depressionsinventar (BDI-II) Revision. Frankfurt, Allemagne: Harcourt Test Services.

Heim, C., \& Nemeroff, C. B. (2001). The role of childhood trauma in the neurobiology of mood and anxiety disorders: Preclinical and clinical studies. Biological Psychiatry, 49, 1023-1039.
Hirschfeld, R. M. (2003). Long-term side effects of SSRIs: Sexual dysfunction and weight gain. Journal of Clinical Psychiatry, 64 (Suppl. 18), 20-24.

Ho, M. S. K., \& Lee, C. W. (2012). Cognitive behaviour therapy versus eye movement desensitization and reprocessing for post-traumatic disorder-Is it all in the homework then? Revue européenne de psychologie appliquée, 62, 253-260.

Hoegberg, G., Pagani, M., Sundin, O., Soares, J., Aeberg-Wistedt, A., Tarnell, B., \& Haellstroem, T. (2007). On treatment with eye movement desensitization and reprocessing of chronic post-traumatic stress disorder in public transportation workers-A randomized controlled trial. Nordic Journal of Psychiatry, 61, 54-61.

Hofmann, A., Hase, M., Liebermann, P., Lehnung, M., Ebner, F., Rost, C., \& Tumani, V. (2014). An EMDR protocol for the treatment of depression. In Luber M. (Ed.), EMDR Scripted Protocols. New York, NY : Springer Publishing.

Holon, S. D., Steward, M. D., \& Strunk, D. (2006). Enduring effects for cognitive behavior therapy in the treatment of depression and anxiety. Annual Review of Psychology, 57, 285-315.

Horwitz, A. V., \& Wakefield, J. C. (2007). The loss of sadness: How psychiatry transformed normal sorrow into depressive disorder. Oxford, United Kingdom: Oxford University Press.

Keller, M. B. (2002). Rationale and options for the longterm treatment of depression. Human Psychopharmacology: Clinical and Experimental, 17 (Suppl. 1), S43-S46.

Kendler, K. S., Hettema, J. M., Butera, F., Gardner, C. O., \& Prescott, C. A. (2003). Life event dimensions of loss, humiliation, entrapment, and danger in the prediction of onsets of major depression and generalized anxiety. Arch Gen Psychiatry, 60(8), 789-796.

Kripalani, S., Yao, X., \& Haynes, R. B. (2007). Interventions to enhance medication adherence in chronic medical conditions: A systematic review. Archive of Internal Medicine, 167, 540-550.

Kupfer, D. J. (1991). Long-term treatment of depression. Journal of Clinical Psychiatry, 52 (Suppl.), 28-34.

Maj, M. (2012). Development and validation of the current concept of major depression. Psychopathology, 45, $135-146$.

Maj, M., Veltro, F., Pirozzi, R., Lobaece, S., \& Magliano, L. (1992). Pattern of recurrence of illness after recovery from an episode of major depression: A prospective study. American Journal of Psychiatry, 149, 795-800.

Manfield, P. (Ed.). (1998). Extending EMDR: A casebook of innovative applications. New York, NY : Norton.

Marquis, J. N. (1991). A report on seventy eight cases treated by eye movement desensitization. Journal of Behaviour Therapy and Experimental Psychiatry, 22, 187-192.

McFarlane, A. C. (2010). The long-term costs of traumatic stress: Intertwined physical and psychological consequences. World Psychiatry, 9, 3-10. 
Murray, C. J., \& Lopez, A. D. (1996). The global burden of disease: A comprehensive assessment of mortality and disability from disease, injuries, and risk factors in 1990 and projected to 2020. Cambridge, MA : Harvard University Press.

Nanni, V., Uher, R., \& Danese, A. (2012). Childhood maltreatment predicts unfavorable course of illness and treatment outcome in depression: A meta-analysis. American Journal of Psychiatry, 169, 141-151.

Nierenberg, A. A., Petersen, T. J., \& Alpert, J. E. (2003). Prevention of relapse and recurrence in depression: The role of long-term pharmacotherapy and psychotherapy. Journal of Clinical Psychiatry, 64, 13-17.

Post, R. M. (1992). Transduction of psychosocial stress into the neurobiology of recurrent affective disorder. American Journal of Psychiatry, 149, 999-1010.

Reid, S., \& Barbui, C. (2010). Long term treatment of depression with selective serotonin reuptake inhibitors and newer antidepressants. British Medical Journal, 340, 752-756.

Risch, N., Herrell, R., Lehner, T., Liang, K. Y., Eaves, L., Hoh, J., (...) Merikanagas, K. R. (2009). Interaction between the serotonin transporter gene (5-HTTLPR), stressful life events, and risk of depression: A metaanalysis. Journal of the American Medical Association, 301, 2462-2471.

Rush, A. J., Trivedi, M. H., Wisniewski, S. R., Nierenberg, A. A., Stewart, J. W., Warden, D.,... Fava, M. (2006). Acute and longer-term outcomes in depressed outpatients requiring one or several treatment steps : A STAR ${ }^{\star} \mathrm{D}$ report. American Journal of Psychiatry, 163, 1905-1917.

Scientific Advisory Board for Psychotherapy (wissenschaftlicher Beirat für Psychotherapie). (2006). Assessment for the scientific recognition of the EMDR method for the treatment of posttraumatic stress disorder. Retrieved from http: / / www.wbpsycho therapie.de

Shapiro, F. (2001). EMDR basic principles and protocols. New York, NY : Norton.

Shapiro, F., \& Maxfield, L. (2002). Eye Movement Desensitization and Reprocessing (EMDR): Information processing in the treatment of trauma. Journal of Clinical Psychology, 58, 933-946.

Shapiro, F., \& Silk-Forrest M. (1997). EMDR, the breakthrough therapy for overcoming anxiety, stress, and trauma. New York, NY : Basic Books.

Shapiro, R. (Ed.). (2009). EMDR solutions 2. New York, NY: Norton.

Sun, T. F., Wu, C. K., \& Chiu, N. M. (2004). Mindfulness meditation training combined with eye movement desensitization and reprocessing in psychotherapy of an elderly patient. Chang Gung Medical Journal, 27, 464-469.

Teicher, M. H., Samson, J. A., Polcari, A., \& Andersen, S. L. (2009). Length of time between onset of childhood sexual abuse and emergence of depression in a young adult sample: A retrospective clinical report. Journal of Clinical Psychiatry, 70, 684-691.

Tinker, R. H., \& Wilson, S. A. (1999). Through the eyes of a child-EMDR with children. New York, NY : Norton.

Uribe, M. E. R., Ramírez, E. O. L., \& Mena, I. J. (2010). Effect of the EMDR psychotherapeutic approach on emotional cognitive processing in patients with depression. The Spanish Journal of Psychology, 13, 396-405.

van der Kolk, B. A., Spinazzola, J., Blaustein, M. E., Hopper, J. W., Hopper, E. K., Korn, D. L., \& Simpson, W. B. (2007). A randomized clinical trial of eye movement desensitization and reprocessing (EMDR), fluoxetine, and pill placebo in the treatment of posttraumatic stress disorder: Treatment effects and long-term maintenance. Journal of Clinical Psychiatry, 68, 37-46.

van Etten, M. L., \& Taylor J. (1998). Comparative efficacy of treatments for post-traumatic stress disorder: A meta-analysis. Clinical and Psychological Psychotherapy, 5 , 126-144.

Vittengl, J. R., Clark, L. A., Dunn, T. W., \& Jarrett, R. B. (2007). Reducing relapse and recurrence in unipolar depression: A comparative meta-analysis of cognitive behavioral therapy's effects. Journal of Consulting and Clinical Psychology, 75, 475-488.

Wilson, S. A., Becker, L. A., \& Tinker, R. H. (1995). Eye movement desensitization and reprocessing (EMDR) treatment for psychologically traumatized individuals. Journal of Consulting and Clinical Psychology, 63, 928-937.

Wilson, S. A., Becker, L. A., \& Tinker, R. A. (1997). Fifteen month follow-up of eye movement desensitization and reprocessing (EMDR) treatment for posttraumatic stress disorder and psychological trauma. Journal of Consulting and Clinical Psychology, 65, 1047-1056.

Wise, L. A., Zierler, S., Krieger, N., \& Harlow, B. L., (2001). Adult onset of major depressive disorder in relation to early life violent victimisation: A case-control study. Lancet, 358, 881-887.

Wittchen, H. U., Zaudig, M., \& Wunderlich, U. (1997). SKID-I, klinisches Interview für DSM Achse 1 Psychische Störungen. Göttigen, Allemagne: Hogrefe.

Merci d'adresser toute correspondance concernant cet article au Dr Arne Hofmann, EMDR Institut Deutschland, Dolmanstrasse 86b, 51427 Bergisch Gladbach, Allemagne. Courriel : arne-hofmann@t-online.de 